\title{
Maximum Power Point of Magnetoelectric Transducer for Wireless Power Transmission
}

\author{
Ali Koteiche ${ }^{1,2}$, Alexis Brenes ${ }^{1}$, Kevin Malleron $^{1}$ and \\ Gerard Sou ${ }^{2}$ \\ 1 ISEP - Institut Supérieur d'Electronique de Paris, 10 rue de Vanves, \\ Issy-les-Moulineaux, 92130-France \\ 2 Sorbonne Université, CNRS, Laboratoire de Génie Electrique et Electronique \\ de Paris (GEEPS), Paris, 75252 \\ E-mail: akoteiche@isep.fr
}

\begin{abstract}
In this contribution, we propose an experimentally-validated systemlevel model of a magnetoelectric transducer and a general methodology for future characterization of similar transducers. This methodology provides insights into the main origin of power losses. An analogy with piezoelectric generators leads to a system-level figure of merit which is strongly impacted by the nonlinear behavior of the transducer. Considering the complexity of the observed nonlinear behavior, we opt for a grey-box model of the dependencies between the transducer characteristics and the operating point. Applying our methodology to an optimized sample shows that nonlinear mechanical losses are the main origin of power dissipation. The maximum transferable power is proven to be up to $61 \%$ lower than the power one would expect from a linear characterization.
\end{abstract}

Keywords: figure of merit, magnetoelectric transducers, power limit, wireless power transmission 


\section{Introduction}

Magnetoelectric (ME) transducers are solutions for wireless power transfer in a variety of applications (energy harvesters, power converters, sensors ...) [1-6]. These transducers may be used for wireless charging of implants, avoiding critical surgical operation. A common way to manufacture these transducers is by bounding layers of magnetostrictive and piezoelectric $(\mathrm{PE})$ materials $[7,8]$. The resulting assembly converts the magnetic energy into mechanical energy (via the magnetostrictive layers) and then into electrical energy (via the PE layer). Like other generators (e.g. PE transducers, photovoltaic cells), a power management $(\mathrm{PM})$ circuit is required between the transducer and the load or storage unit to ensure optimal energy transmission [9-16]. The design of an efficient PM electrical interface relies on the generator characteristics. For instance, previous literature defines the most relevant system-level parameters of a linear piezoelectric generator for the design of an electrical interface [9]. Among them, a so-called figure of merit (FOM) is determined. It was proven to be a crucial criterion for impedance matching. This FOM is the product of two material-related parameters: the quality factor $Q$ and the expedient electromechanical coefficient $k_{m}^{2}[17,18]$. The corresponding power converted by the $\mathrm{PE}$ transducer is known to be bounded, the upper bound being sometimes called theoretical power limit $\left(P_{\text {lim }}\right)[19,20]$.

Despite the similarities between the two systems ( $\mathrm{PE}$ and $\mathrm{ME}$ ), the power limitation and FOM of a ME transducer have not been determined yet. Therefore, we propose an experimentally-validated system-level model of a magnetoelectric transducer and a general methodology for the characterization of such transducers.

This contribution is divided into three sections. Section 2 is dedicated to a nonlinear single-degree-offreedom (SDOF) model and its main parameters of interest. We determine the theoretical power limit and the figure of merit of a ME transducer. Then, in section 3, we propose a method to characterize the generator and validate it experimentally. Finally, the experimental results are analyzed in terms of power losses, transferable power and impedance matching condition in section 4 .

\section{Theoretical study}

\subsection{System-level model of a magnetoelectric transducer}

The ME transducer is placed under a static magnetic field (generated by a permanent magnet). A variable magnetic field at angular frequency $\omega$ is generated by a current $i_{\text {in }}=i_{0} \sin \omega t$ flowing through a coil. Since the dimensions of the ME transducer are very small with respect to the wavelength of the $\mathrm{AC}$ magnetic field, the latter is uniform inside the sample. The SDOF model of the magnetoelectric transducer (ME) under such conditions is given in Fig. 1. In this model, $C_{p}$ is the capacitance of the piezoelectric element. The leak resistance $r_{p}$, in parallel with $C_{p}$, accounts for the electrical losses inside the piezoelectric layer. The piezoelectric output current is written $i$ and the piezoelectric voltage $u$. The mechanical model is made of an effective mass $M$ of motion $x$ with respect to the base, suspended by a spring of stiffness $K(x)$. A previous study has proven that the system exhibits a nonlinear stiffness [21]. For that reason, we assume that the stiffness is given by (1).

$K(x)=K_{l i n}+K_{N L}(x)$

With this definition, $K_{\text {lin }}$ corresponds to the stiffness of the resonator for a very low vibration amplitude and $K_{N L}(x)$ to the displacement-dependent stiffness of the system. The natural angular (shortcircuit) frequency is $\omega_{0}(x)=\sqrt{K(x) / M}$ and the natural frequency is $f_{0}(x)=\omega_{0}(x) / 2 \pi$. We also define the natural angular frequency $\omega_{0_{l i n}}=\sqrt{K_{l i n} / M}$ in linear regime. The factor $\beta$ (N/A) accounts for the bidirectional magneto-mechanical coupling (MMC) and the factor $\alpha(\mathrm{N} / \mathrm{V})$ for the bidirectional electromechanical coupling (EMC). One may expect small variations of $\alpha$ with the motion $x$ [22-24] but our experimental results (see section 3) allow us to neglect the variations of $\alpha$. The mechanical and magnetic losses in the circuit are modeled by a nonlinear damper (2).

$c(x, d x / d t)=c_{\text {lin }}+c_{N L}(x, d x / d t)$

With this definition, $c_{l i n}$ accounts for the damping of the resonator at a very low vibration amplitude and $c_{N L}\left(x, \frac{d x}{d t}\right)$ for all the potential sources of nonlinear damping.

Many physical phenomena may be the source of nonlinear damping $[22,25]$. Among them, one may expect structural damping when the elastic material is imperfect and/or due to slip or friction or numerous other physical phenomena [26]. Based on these assumptions, the motion of mass $M$, is governed by (3) and (4).

$M \frac{d^{2} x}{d t^{2}}+c\left(x, \frac{d x}{d t}\right) \frac{d x}{d t}+K(x) x+\alpha u-\beta i_{\text {in }}=0$,
$\frac{u}{r_{\text {load }}}=\alpha \frac{d x}{d t}-C_{p} \frac{d u}{d t}-\frac{u}{r_{p}}$

The corresponding quality factor is defined by (5) where $Q_{l i n}$ and $Q_{N L}\left(x, \frac{d x}{d t}\right)$ are given in (6) and (7). 


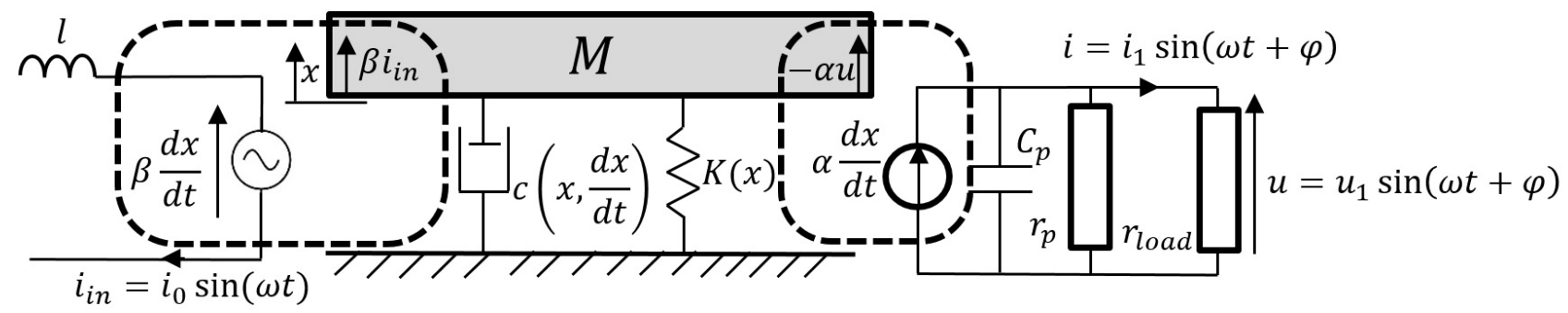

Figure 1. Magneto-electromechanical model of the ME transducer.

$$
\begin{aligned}
& \frac{1}{Q\left(x, \frac{d x}{d t}\right)}=\frac{1}{Q_{l i n}}+\frac{1}{Q_{N L}\left(x, \frac{d x}{d t}\right)} \\
& Q_{l i n}=\frac{M \omega_{0_{l i n}}}{c_{l i n}} \\
& Q_{N L}\left(x, \frac{d x}{d t}\right)=\frac{M \omega_{0}(x)}{c_{N L}\left(x, \frac{d x}{d t}\right)}
\end{aligned}
$$

Unfortunately the aforementioned parameters are dependent on the fabrication process of the sample and the motion amplitude. Hence, no analytical expression is readily available. This justifies the choice of a greybox model detailed in the following sections.

\subsection{Figure of merit and maximum power point of a ME transducer}

2.2.1. Linearized case of a leakless transducer In order to determine the maximum transferable power, the piezoelectric layer is assumed to be leakless $\left(r_{p}=\right.$ $\infty)$. Since the parameters depend on the mechanical motion, the system is linearized in the vicinity of an amplitude $x_{0}$. In such case, the behavior of the ME system becomes (8) and (9). The linearized quality factor then verifies (10).

$M \frac{d^{2} x}{d t^{2}}+c\left(x_{0}, \frac{d x}{d t}\left(x_{0}\right)\right) \frac{d x}{d t}+K\left(x_{0}\right) x+\alpha u-\beta i_{i n}=0,(8)$

$$
\frac{u}{r_{\text {load }}}=\alpha \frac{d x}{d t}-C_{p} \frac{d u}{d t}
$$

$\frac{1}{Q\left(x_{0}\right)}=\frac{1}{Q_{\text {lin }}}+\frac{1}{Q_{N L}\left(x_{0}, \frac{d x}{d t}\left(x_{0}\right)\right)}$

Based on the notations given in Table 1, we obtain (11) and (12), which bring out the expedient electromechanical coupling coefficient $k_{m}^{2}[17,18]$ given in (13) and an expedient magnetoelectric coupling coefficient $B_{m}$ (14). In (12), $R_{\text {load }}$ stands for the normalized resistance $R_{\text {load }}=r_{\text {load }} C_{p} \omega_{0}\left(X_{0}\right)$.

$$
\begin{aligned}
& \ddot{X}+\frac{\dot{X}}{Q\left(X_{0}\right)}+X+U-B_{m} I_{\text {in }}=0, \\
& \frac{U}{R_{\text {load }}}=k_{m}^{2} \dot{X}-\dot{U},
\end{aligned}
$$

$$
\begin{aligned}
k_{m}^{2}\left(X_{0}\right) & =\frac{\alpha^{2}}{K\left(X_{0}\right) C_{p}} \\
B_{m}\left(X_{0}\right) & =\frac{\beta C_{p} \omega_{0}\left(X_{0}\right)}{\alpha}
\end{aligned}
$$

Table 1. Notations

\begin{tabular}{|c|c|c|}
\hline Variable & Quantity (unit) & Normalized variable \\
\hline \hline$\omega$ & Vibration angular frequency $\left(\right.$ rad.s $\left.{ }^{-1}\right)$ & $\Omega=\frac{\omega}{\omega}$ \\
\hline$x$ & Displacement of the resonator $(m)$ & $X=\frac{x M \omega_{0}^{2}}{\beta i_{0}}$ \\
\hline$i_{i n}$ & Input current $(A)$ & $I_{i n}=\frac{i_{i n} k_{m}^{2} M \omega_{0}}{\alpha \beta i_{0}}$ \\
\hline$i$ & Output current $(A)$ & $I=\frac{i k_{m}^{2} M \omega_{0}}{\alpha \beta i_{0}}$ \\
\hline$u$ & Output voltage $(V)$ & $U=\frac{u \alpha}{\beta i_{0}}$ \\
\hline
\end{tabular}

For the sake of readability, in this subsection, we will omit the parentheses (e.g. write $Q, \omega_{0}$ instead of $\left.Q\left(X_{0}\right), \omega_{0}\left(X_{0}\right)\right)$.

In order to reach impedance matching, some specific operating conditions must be fulfilled. Writing $u=u_{1} \sin (\omega t+\phi)$, the transfer function between the first-harmonic voltage and the first-harmonic motion can be written (15) [19].

$\frac{\underline{\mathrm{U}}_{1}}{\underline{\mathrm{X}}_{1}}=k_{m}^{2}\left(U_{p}(\Omega)+j U_{q}(\Omega)\right)$,

The two conditions to guarantee impedance matching are (16) and (17).

$\Omega^{2}=1+k_{m}^{2} U_{p}(\Omega)$,

$k_{m}^{2} Q=-\frac{\Omega}{U_{q}(\Omega)}$

For a PE transducer, a well-known systemlevel FOM is the product $k_{m}^{2} Q$ appearing in (17). Depending on this FOM, optimal power transmission may or may not be achieved by a circuit at a given frequency. It all depends on the mathematical possibility to verify simultaneously (16) and (17) [19]. Since the rules (16) and (17) are the same for PE and ME transducers, $k_{m}^{2} Q$ is also a relevant FOM for magnetoelectric systems. 
2.2.2. Maximum power transmission for a $M E$ transducer In the literature, the theoretical limitation to the power transferred by a PE transducer when subject to a given acceleration $\gamma=\ddot{y}=\gamma_{m} \sin (\omega t)$ is given by (18) [27]. This power limit can be achieved with a relevant power management circuit between the transducer and the load, guaranteeing impedance matching.

$P_{l i m_{P E}}=\frac{M \gamma_{m}^{2} Q}{8 \omega_{0}}$

A significant difference between the $\mathrm{PE}$ and the ME transducers is that the latter is not an inertial system, which means that its actuation force $\beta i_{i n}$ (see (3)) is not proportional to its mass (whereas it is $\gamma M$ for the PE transducer (20)). Another significant difference lies in the fact that the parameters $k_{m}^{2}$ and $Q$ are the result of the complex magnetic, mechanical and electrical interaction between the piezoelectric and the magnetostrictive layers. Our experiments prove that both parameters differ from the coupling coefficient and quality factor of the piezoelectric sample alone. No analytical expression is available for such a system. However, considering the analogy between the PE and ME models, the determination of the power limit for a ME transducer actuated by an AC input current $i_{\text {in }}=i_{0} \sin (\omega t)$ can be obtained with the substitution (19).

$M \gamma_{m} \leftrightarrow \beta i_{0}$,

This substitution leads to the maximum theoretical power $P_{l i m_{M E}}(21)$. Contrary to PE transducers, the power limit of ME systems is not proportional to the effective mass $M$, but to the square of the expedient magnetoelectric coefficient $B_{m}$. It is also proportional to the transducer FOM $k_{m}^{2} Q$, which is a significant difference with the $\mathrm{PE}$ case.

$$
\begin{aligned}
& M \frac{d^{2} x}{d t^{2}}+c \frac{d x}{d t}+K x+\alpha u-M \gamma=0, \\
& P_{l i m_{M E}}=\frac{B_{m}^{2} k_{m}^{2} Q i_{0}^{2}}{8 C_{p} \omega_{0}}
\end{aligned}
$$

All these considerations about the FOM and the maximum power transmission of a $\mathrm{ME}$ transducer highlight the importance of the system-level parameters $k_{m}^{2}, Q, B_{m}$ and $C_{p}$. In the following section, we validate this model experimentally and quantify the nonlinear behavior of our ME transducer. We determine the impact of this nonlinear behavior on the maximum power and FOM of the generator.

\section{Experimental study}

\subsection{Experimental setup}

Fig. 2 shows a picture of the ME sample: two magnetostrictive layers (Terfenol-D longitudinally-polarized) of the same dimensions $(10 \mathrm{~mm} \times 14 \mathrm{~mm} \times 1 \mathrm{~mm})$ glued to a piezoelectric plate (P51 longitudinally-polarized) $(10 \mathrm{~mm} \times 20 \mathrm{~mm} \times 1 \mathrm{~mm})$. This sample has been optimized based on a previous work [28]. A wire is welded at each side of the piezoelectric sample. The sample is exposed to an optimal DC magnetic field $H_{D C}=40 \times 10^{3} \mathrm{~A} / \mathrm{m}$, chosen to maximize the opencircuit voltage via a permanent magnet arrangement. The additional AC magnetic field is generated by the excitation coil connected to an Agilent 33220A generator. We perform frequency sweeps from $f_{\text {min }}=65 \mathrm{kHz}$ to $f_{\max }=72 \mathrm{kHz}$ for several loads around $r_{\text {load }}=600 \Omega$ close to the optimal resistance. A picture and a schematic of the setup are given in Fig. 3 and Fig. 4.

As described in the theoretical part, the damping coefficient $c\left(x, \frac{d x}{d t}\right)$ and the stiffness $K(x)$ are expected to change when the amplitude $x_{m}$ of the displacement $x$ varies. Hence, controlling the amplitude of the displacement would ensure that the system parameters do not vary significantly during each sweep. Unfortunately, controlling the mechanical motion without dedicated instruments like a laser vibrometer is complex. However, the motion amplitude $x_{m}$ is proportional to the amplitude $i_{1}$ of the output current as long as the load resistance is constant (see (22) obtained from (9)). In (22), $\omega_{m}=\pi\left(f_{\max }+f_{\min }\right)$ is the center of the frequency interval. Equation (22) assumes that the resonator is characterized on a narrow frequency range due to its high Q-factor. Hence, controlling the amplitude $i_{1}$ of the output current and the load $r_{\text {load }}$ guarantees that the amplitude of the mechanical motion remains constant during each sweep.

$x_{m}=\frac{i_{1}}{\alpha \omega} \sqrt{1+\frac{\omega^{2}}{\omega_{0}^{2}} R_{\text {load }}^{2}} \simeq \frac{i_{1}}{\alpha \omega_{m}} \sqrt{1+\frac{\omega_{m}^{2}}{\omega_{0}^{2}} R_{\text {load }}^{2}}$

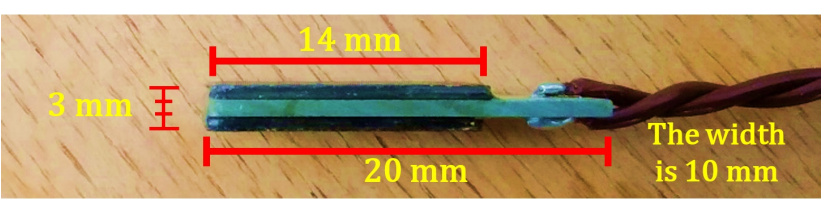

Figure 2. ME sample: a PE layer (light green) between two magnetostrictive layers (dark). Each one is glued to one side of the PE plate.

\subsection{Preliminary admittance measurements}

In order to determine the capacitance $C_{p}$ and the coupling coefficient $k_{m}^{2}$ of the ME sample, we performed admittance measurements (with Zurich Instruments MFIA Impedance Analyzer) under $H_{D C}$ in the absence of any AC magnetic field. An example is given in Fig. 5 where $f=\frac{\omega}{2 \pi}$. The sweep duration is 20 s and the sweep step is $100 \mathrm{~Hz}$. Measurements at 


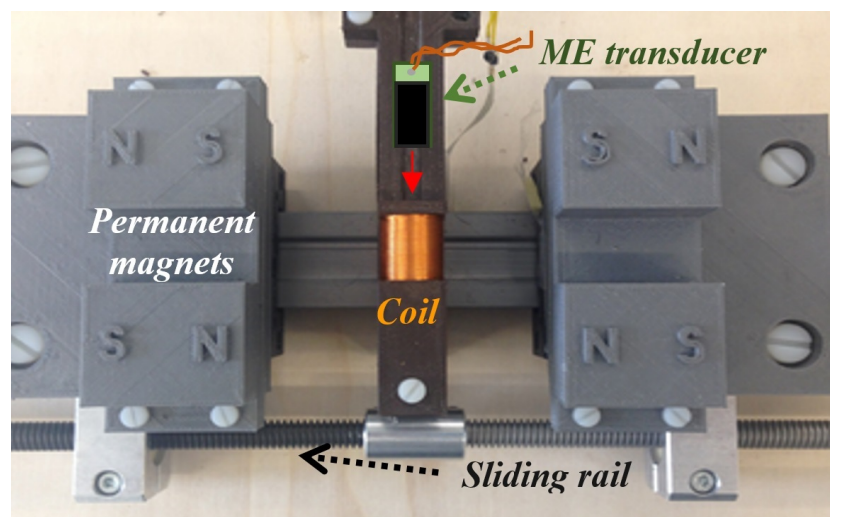

Figure 3. Test bench made of an excitation coil (100 turns, $18 \mathrm{~mm}$ of diameter, $0.1 \mathrm{~mm} \varnothing \mathrm{wire}$ ), a sliding rail to adjust the permanent magnet position and to reach the optimal static magnetic field of $40 \times 10^{3} \mathrm{~A} / \mathrm{m}$. The ME transducer is put inside the sample holder surrounded by the coil.

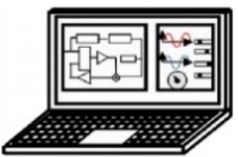

- PID voltage control

- Voltage acquisitions

Via Matlab

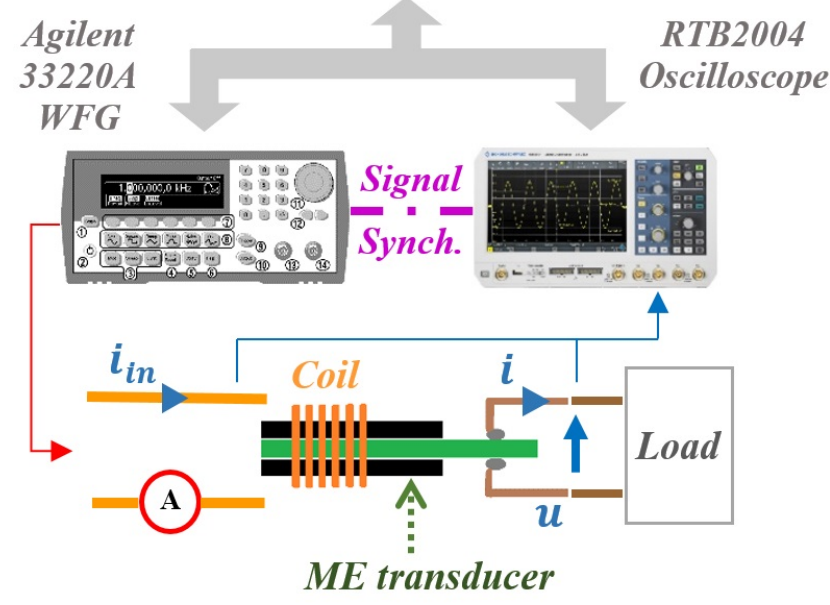

Figure 4. Schematic of the experimental setup.

other amplitudes from $0.1 \mathrm{~V}$ to $3 \mathrm{~V}$ have shown that neither $C_{p}$ nor $k_{m}^{2}$ vary significantly. We found that $C_{p} \simeq 2 n F$ and $k_{m}^{2} \simeq 5.48 \times 10^{-2}$.

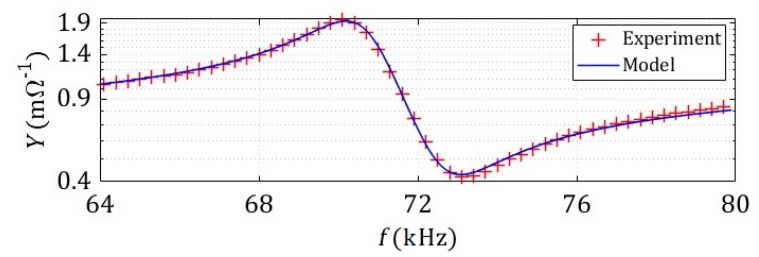

Figure 5. Admittance measurements $\left(u_{1}=1.5 \mathrm{~V}\right)$

\subsection{Experimental results}

In the series of measurements, the amplitude of the output current is regulated at different levels from $0.28 \mathrm{~mA}$ to $2.55 \mathrm{~mA}$. For this range of $i_{\text {out }}$, the amplitude $i_{0}$ of the input current $i_{\text {in }}$ varies from $2 m A$ to $90 \mathrm{~mA}$. We perform the measurements for 8 loads between $270 \Omega$ and $2200 \Omega$. Parameters are identified from a nonlinear least-squares fitting procedure on the transfer function $u_{1} / i_{0}$ which can be obtained from (11) and (12) expressed in the frequency domain either numerically or analytically. The corresponding analytical formula is given in (23) where $d_{1}(\Omega)=$ $2 k_{m}^{2} Q R_{\text {load }}+1+R_{\text {load }}^{2} \Omega^{2}$ and $d_{2}(\Omega)=\Omega^{2}-2+R_{\text {load }}^{2}(1+$ $\left.k_{m}^{2}-\Omega^{2}\right)^{2}$.

Fig. 6 illustrates three examples at $i_{1}=0.28 \mathrm{~mA}$, $i_{1}=1.41 \mathrm{~mA}$ and $i_{1}=2.55 \mathrm{~mA}$. The corresponding estimated parameters are reported in Fig. 7.

$$
\frac{u_{1}}{i_{0}}(\Omega)=\frac{B_{m} k_{m}^{2} R_{\text {load }} \Omega}{\omega_{0} C_{p} \sqrt{\frac{\Omega^{2}}{Q^{2}} d_{1}(\Omega)+1+\Omega^{2} d_{2}(\Omega)}}
$$

For each load, we observe that:

- the quality factor $Q$ and the natural frequency $f_{0}$ decrease,

- the expedient magnetoelectric coeficient $B_{m}$ slightly rises

when the amplitude $i_{1}$ of the piezoelectric current $i$ increases.

\subsection{Further analysis and validation}

To analyze further the results given in section 3.3, we quantify the evolution of the parameters with the output current. To that purpose, we start by fitting an empirical law on the parameters $Q\left(i_{1}\right), f_{0}\left(i_{1}\right)$ and $B_{m}\left(i_{1}\right)$. The corresponding laws are given in (24), (25) and (26) in which $i_{\min }=0.28 \mathrm{~mA}$ is the minimum amplitude of the output current set in our experiments. Since each $r_{\text {load }}$ leads to a different mechanical motion amplitude (see (22)), the coefficients of the empirical law must be adjusted depending on the resistive load. The values of the parameters in the empirical laws for the resistances tested in this setup are given in Tables 2, 3 and 4 . The evolution of $f_{0}^{2}$ corresponds to a spring softening effect, preponderant at low amplitude, and a slight hardening effect balancing the softening effect as the motion amplitude increases. The evolution of $B_{m}$ shows that the magnetostrictive coefficient $\beta$ is proportional to the output current. Lastly, (24) combined with (22) shows that the energy losses rise significantly with the amplitude of the mechanical motion. These results provide the quality factor $Q_{\text {lin }}(27)$, the natural frequency $f_{0_{\text {lin }}}(28)$ and the magnetoelectric coefficient $B_{m_{l i n}}(29)$ in linear 

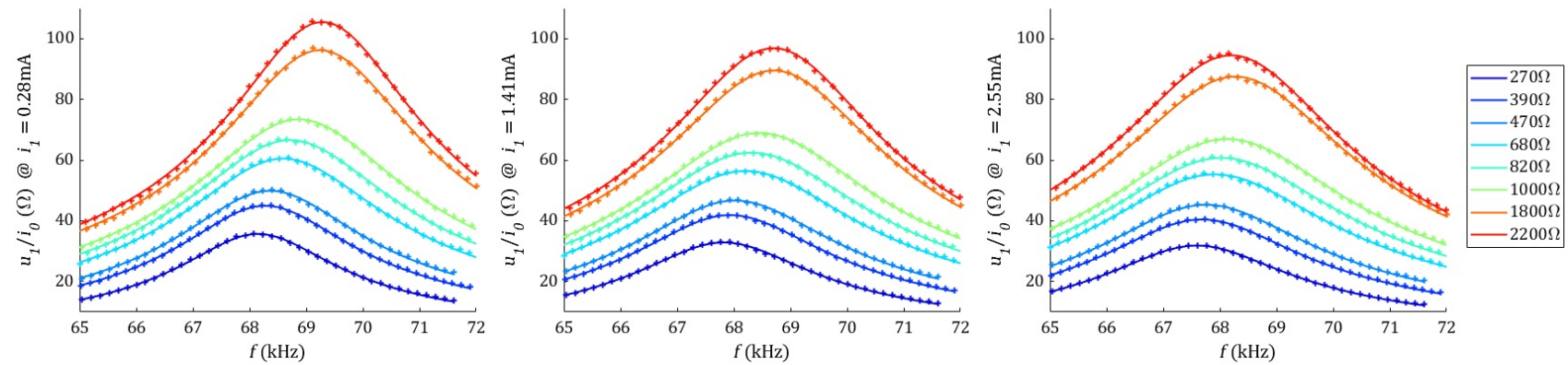

Figure 6. Model identification: experimental frequency responses $u_{1} / i_{0}$ for a regulated amplitude of the output current (left: $i_{1}=0.28 \mathrm{~mA}$, middle: $i_{1}=1.41 \mathrm{~mA}$, right: $\left.i_{1}=2.55 \mathrm{~mA}\right)$. Crosses: measurements. Solid lines: model. From blue to red: $r_{\text {load }}=270 \Omega$ to $r_{\text {load }}=2200 \Omega$.

regime (at low motion amplitude). The corresponding standard deviation $\sigma$ is also indicated.

$\frac{1}{Q\left(i_{1}\right)}=\frac{1}{Q_{\text {lin }}}+p_{1}\left(\frac{i_{1}}{i_{\min }}\right)^{q}$,

$f_{0}^{2}\left(i_{1}\right)=f_{0_{l i n}}^{2}\left[1-\nu_{1} \frac{i_{1}}{i_{\min }}+\nu_{2}\left(\frac{i_{1}}{i_{\min }}\right)^{2}\right]$,

$B_{m}\left(i_{1}\right)=B_{m_{l i n}}\left[1+b_{1} \frac{i_{1}}{i_{\text {min }}}\right]$,

Table 2. Empirical law of $Q$

\begin{tabular}{|c|c|c|c|}
\hline$r_{\text {load }}(\Omega)$ & $Q_{\text {lin }}$ & $q\left(\times 10^{-1}\right)$ & $p_{1}\left(\times 10^{-2}\right)$ \\
\hline 270 & 47.9 & 3.484 & 0.692 \\
\hline 390 & 47.8 & 3.526 & 0.695 \\
\hline 470 & 49.0 & 3.598 & 0.702 \\
\hline 680 & 56.1 & 3.012 & 0.929 \\
\hline 820 & 53.4 & 3.282 & 0.820 \\
\hline 1000 & 57.9 & 3.152 & 0.883 \\
\hline 1800 & 50.8 & 4.166 & 0.620 \\
\hline 2200 & 46.4 & 4.879 & 0.502 \\
\hline
\end{tabular}

Table 3. Empirical law of $f_{0}^{2}$

\begin{tabular}{|c|c|c|c|}
\hline$r_{\text {load }}(\Omega)$ & $f_{0_{\text {lin }}}(\mathrm{kHz})$ & $\nu_{1}\left(\times 10^{-3}\right)$ & $\nu_{2}\left(\times 10^{-4}\right)$ \\
\hline 270 & 68.15 & 2.84 & 0.83 \\
\hline 390 & 68.19 & 2.90 & 0.77 \\
\hline 470 & 68.21 & 3.04 & 0.82 \\
\hline 680 & 68.22 & 3.15 & 0.74 \\
\hline 820 & 68.22 & 3.55 & 1.10 \\
\hline 1000 & 68.20 & 3.51 & 0.93 \\
\hline 1800 & 68.08 & 4.40 & 1.08 \\
\hline 2200 & 68.00 & 4.47 & 0.65 \\
\hline
\end{tabular}

$Q_{\text {lin }}=\frac{2 \pi M f_{0_{l i n}}}{c_{l i n}}=51.2 \quad(\sigma=4.2)$,
Table 4. Empirical law of $B_{m}$

\begin{tabular}{|c|c|c|}
\hline$r_{\text {load }}(\Omega)$ & $B_{m_{\text {lin }}}\left(\times 10^{-2}\right)$ & $b_{1}\left(\times 10^{-3}\right)$ \\
\hline 270 & 9.86 & 7.96 \\
\hline 390 & 9.83 & 7.06 \\
\hline 470 & 9.69 & 7.60 \\
\hline 680 & 9.57 & 7.66 \\
\hline 820 & 9.52 & 7.23 \\
\hline 1000 & 9.39 & 6.85 \\
\hline 1800 & 9.05 & 8.63 \\
\hline 2200 & 9.02 & 9.14 \\
\hline
\end{tabular}

$$
\begin{aligned}
& f_{0_{l i n}}=\frac{1}{2 \pi} \sqrt{\frac{K_{l i n}}{M}}=68160 H z \quad(\sigma=81 H z), \\
& B_{m_{l i n}}=9.5 \times 10^{-2} \quad\left(\sigma=3.2 \times 10^{-3}\right),
\end{aligned}
$$

Ultimate validation of our model requires to check its predictivity. To that purpose, we perform another series of measurements where the amplitude $i_{0}$ of the input current is regulated, instead of the amplitude $i_{1}$ of the output current. If our model is accurate (i.e. if the parameters mainly depend on the output), we should be able to predict the evolution of $i_{1}$ during the frequency sweeps at $i_{0}$ constant by taking into account the parameter variations identified in Fig. 7. We report in Fig. 8, the predicted evolution of the output current at three regulated amplitudes $i_{0}=$ $11.3 \mathrm{~mA}, i_{0}=19.8 \mathrm{~mA}$ and $i_{0}=35.4 \mathrm{~mA}$ for several loads, along with the experimental measurements. The agreement between the experiments and the predicted behavior is very good. If the parameters did mostly depend on the AC magnetic field (i.e. on the input current), the predicted behavior would be far from the observations. Despite the good correspondence, we observe a small deviation between the predictions and the measurements which implies that the parameters may also slightly depend on the input current.

Now that the model has been validated, interesting conclusions can be drawn from our characterization. A first set of conclusions relates to the main 

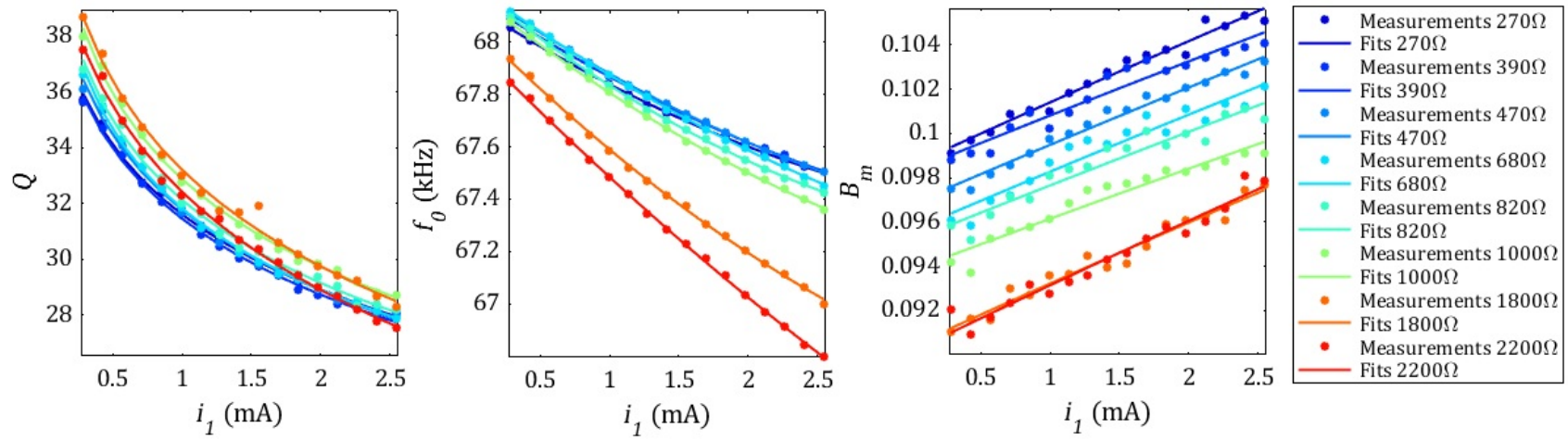

Figure 7. System parameters as a function of the output current $i_{1}$ for several resistive loads. Left: quality factor $Q$, middle: natural frequency $f_{0}$, right: expedient magnetoelectric coefficient $B_{m}$ (Dots : Measurements. Solid lines: Fits).
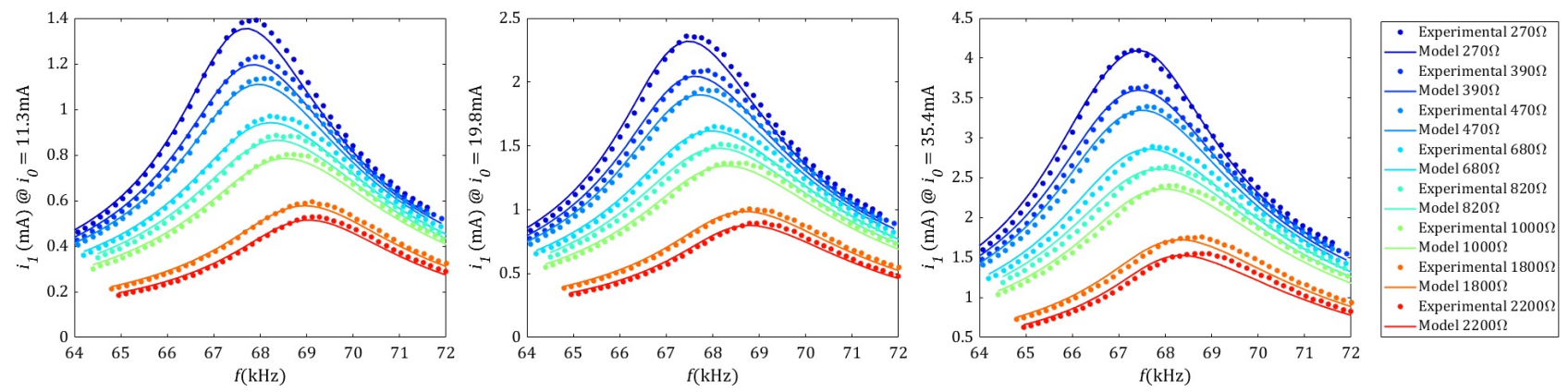

Figure 8. Model validation: experimental frequency responses $u_{1} / i_{0}$ for a regulated amplitude of the input current (left: $i_{0}=11.3 \mathrm{~mA}$, middle: $i_{0}=19.8 \mathrm{~mA}$, right: $i_{0}=35.4 \mathrm{~mA}$ ). Dots: measurements. Solid lines: model. From blue to red: $r_{l o a d}=270 \Omega$ to $r_{\text {load }}=2200 \Omega$.

physical origin of performance degradation in our device. The second set deals with the consequences of the system nonlinear behavior on the circuit design and on the transferable power. These discussions are the subjects of section 4 .

\section{Discussion}

\subsection{About the physical origin of power losses}

Specific physical phenomena lie in the evolution of the estimated quality factor. In terms of energy losses, the overall system can be decomposed into a resistive part in the RLC-series equivalent model of the resonator and a parallel resistance $r_{p}$. In our model, the quality factor $Q$ accounts for all the energy losses (magnetic and/or mechanical) except the electrical losses related to leak currents.

4.1.1. Electrical losses In section 2.1, we modelled the electrical losses with the leak resistance $r_{p}$ and stated that it is often neglected in theoretical as well as in experimental studies. In our case, we measured the parallel resistance $r_{p}$ and it is so large that we are not able to determine its exact value. Our measurements suggest that it is superior to $2 M \Omega$, which is larger than $r_{\text {load }}$ by 3 orders of magnitude. For this reason, we can safely assume that the electrical losses are negligible and are not the cause of the observed variations of the parameters. In particular, they cannot explain the decrease of the quality factor when the current increases.

4.1.2. Magnetic losses The origin of magnetic losses is mainly related to eddy current in the magnetostrictive layer $[29,30]$. Eddy currents are expected to increase when the AC magnetic fields becomes larger. In section 3.3 , we have proven that $Q$ may reasonably be assumed to be independent of $i_{0}$ and dependent mainly on $i_{1}$. Hence, in our setup, the magnetic losses may be neglected compared to the (nonlinear) mechanical losses.

4.1.3. Mechanical losses Since other origins have been eliminated, the preponderant energy losses in our system are mechanical. As stated in section 2.1 , a damping coefficient that is dependent on the displacement $x$ and/or the velocity $d x / d t[23$, $25]$ is typically related to mechanical dissipation. 

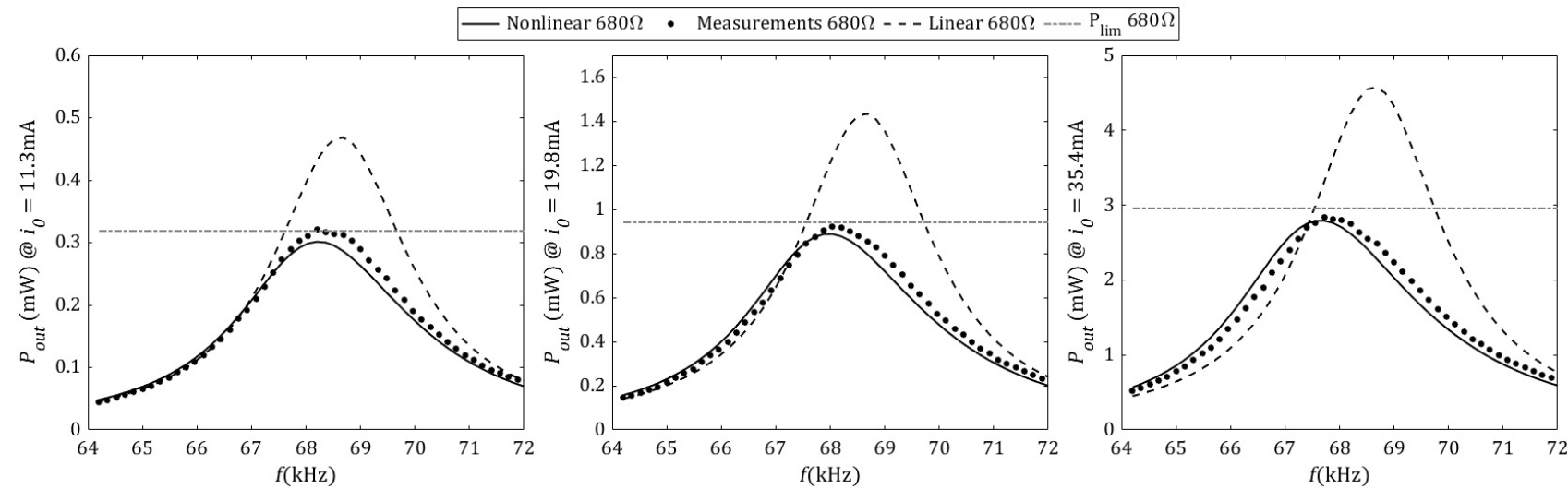

Figure 9. Power vs. frequency responses for a regulated amplitude of the input current (left: $i_{0}=11.3 m A$, middle: $i_{0}=19.8 m A$, right: $i_{0}=35.4 m A$ ). Dots : Experimental measurements. Solid lines : Responses reconstructed based on the characterization at regulated output currents. Dashed lines : Responses expected from a linear model. Dash-dot line (gray): power limit of the ME transducer.

Measurements performed on the PE element before gluing to the magnetostrictive layers have shown that the PE layer alone behaves linearly. Significant discrepancies appear after gluing the PE layer to the magnetostrictive layers. For this reason, we suspect the losses to come from dissipation inside the glue and/or inside the magnetostrictive layers.

\subsection{Impact of parameter variations on circuit design}

The transferable power as well as the impedance matching condition are affected by the nonlinear behavior of the system through the variation of $Q$ (see section 2.1). One may wonder about the consequences of the previous characterization on the output power when the input current is constant, which is the most common and practical situation. In such case, we have demonstrated that a good way to determine the system response is to characterize it at several levels of output current and then reconstruct the response for a given input current. This is what we did in Fig. 9, where we plot the power vs. frequency curves for the optimal load $(680 \Omega)$ for regulated input currents of $11.3 m A, 19.8 m A$ and $35.4 m A$. We compare the result to what one would expect from a characterization of a linear system. This graph highlights how the peak power measured at $11.3 \mathrm{~mA}$ would be overestimated by around $48 \%$ with the linear assumption. Since the load has been optimally chosen, the experimental peak power reaches the power limit $P_{\text {lim }}(21)$ predicted from the identified parameters. Even though this discussion remains qualitatively valid for other input levels, the situation is even more complex that the exact conclusions quantitatively depend on the considered levels of current. At higher input levels, the same conclusions hold. However, the discrepancy between the linear assumption and the experimentally-validated model increases. For instance, results at $i_{0}=35.4 \mathrm{~mA}$ lead to an overestimation of $61 \%$, instead of $48 \%$ previously (see Fig. 9).

The nonlinearity of the transducer also affects the value of the optimal load which linear theory would expect to be $350 \Omega$ [31].

All these considerations thus have a huge impact on further circuit design.

\section{Conclusion}

In this paper, we studied a ME transducer using a SDOF model. We proposed a methodology to identify the model parameters and noticed that, for the current levels achieved in our setup, the most consistent result is obtained when regulating the output current. This is explained by the dependency between the parameters of interest and the mechanical motion.

In particular, the evolution of the quality factor proves that the main origin of energy losses in our device lies in a phenomenon that is dependent on the mechanical vibration amplitude. More importantly, the results invalidate the assumption of predominant magnetic losses. In terms of transferable power, we quantified the impact of the nonlinear behavior on the maximum power achievable by our transducer. We also introduced the figure of merit of the ME transducer and demonstrated that the maximum transferable power is a function of the model parameters and of the input current. We showed that trying to transfer more power by increasing the input current may not be as efficient as expected due to increasing mechanical energy losses.

In terms of analysis of the physical phenomena which take place in magnetoelectric assemblies, this study paves the way to future works to minimize nonlinear effects and energy losses and to maximize 
the amount of transferred power. In terms of electrical design, it quantifies the constraints that must be taken into account before implementation. Further investigations will focus on these two aspects. In addition, analyzing the impact of a non-sinusoidal input on the system behavior (e.g. in power converters) is the subject of ongoing work.

\section{References}

[1] Jingen $\mathrm{Wu}$, Zhongqiang $\mathrm{Hu}$, Xiangyu Gao, Miaomiao Cheng, Xinger Zhao, Wei Su, Zhiguang Wang, Ziyao Zhou, Shuxiang Dong, and Ming Liu. A magnetoelectric compass for in-plane ac magnetic field detection. IEEE Transactions on Industrial Electronics, 2020.

[2] Chung Ming Leung, Jiefang Li, D Viehland, and X Zhuang. A review on applications of magnetoelectric composites: from heterostructural uncooled magnetic sensors, energy harvesters to highly efficient power converters. Journal of Physics D: Applied Physics, 51(26):263002, 2018.

[3] Fumio Narita and Marina Fox. A Review on Piezoelectric, Magnetostrictive, and Magnetoelectric Materials and Device Technologies for Energy Harvesting Applications. Advanced Engineering Materials, 20(5):1700743, May 2018.

[4] RV Petrov, MI Bichurin, VS Leontiev, N Kolesnikov, ST Bozhkov, IK Milenov, G Tonkov, V Boinovski, L Lyubomirov, and PT Bozhkov. Researching the characteristics of the magnetoelectric position sensor for automotive application. In 2018 20th International Symposium on Electrical Apparatus and Technologies (SIELA), pages 1-6. IEEE, 2018.

[5] Haosen Wang, Liyan Qu, Wei Qiao, and Bo Liu. Magnetic flux valve: A magnetoelectric materials-based device for conversion and control of electric power. IEEE Transactions on Industrial Electronics, 64(3):2451-2458, 2016.

[6] NH Duc, BD Tu, NT Ngoc, VD Lap, and DT Huong Giang. Metglas/pzt-magnetoelectric 2-d geomagnetic device for computing precise angular position. IEEE Transactions on Magnetics, 49(8):4839-4842, 2013.

[7] Tyrel Rupp, Binh Duc Truong, Shane Williams, and Shad Roundy. Magnetoelectric transducer designs for use as wireless power receivers in wearable and implantable applications. Materials, 12(3):512, 2019.

[8] Wei He, Ping Li, Yumei Wen, Jitao Zhang, Aichao Yang, and Caijiang Lu. A noncontact magnetoelectric generator for energy harvesting from power lines. IEEE Transactions on Magnetics, 50(11):1-4, 2014.

[9] Alexis Brenes, Elie Lefeuvre, Seonho Seok, and ChanSei Yoo. Tunable unipolar synchronized electric charge extraction strategy for piezoelectric energy harvesting. Journal of Intelligent Material Systems and Structures, 30(11):1629-1638, July 2019.

[10] Huakang Xia, Yinshui Xia, Yidie Ye, Libo Qian, Ge Shi, and Renwen Chen. Analysis and Simulation of Synchronous Electric Charge Partial Extraction Technique for Efficient Piezoelectric Energy Harvesting. IEEE Sensors Journal, 18(15):6235-6244, August 2018.

[11] E Lefeuvre, A Badel, A Brenes, S Seok, M Woytasik, and C-S Yoo. Analysis of piezoelectric energy harvesting system with tunable SECE interface. Smart Materials and Structures, 26(3):035065, March 2017.

[12] Yipeng Wu, Adrien Badel, Fabien Formosa, Weiqun Liu, and Amen E Agbossou. Piezoelectric vibration energy harvesting by optimized synchronous electric charge extraction. Journal of Intelligent Material Systems and Structures, 24(12):1445-1458, August 2013.

[13] Hui Shao, Chi-Ying Tsui, and Wing-Hung Ki. The design of a micro power management system for applications using photovoltaic cells with the maximum output power control. IEEE Transactions on Very Large Scale Integration (VLSI) Systems, 17(8):1138-1142, 2009.

[14] Mohamed Orabi, Fatma Hilmy, Ahmed Shawky, Jaber A Abu Qahouq, El-Sayed Hasaneen, and Eman Gomaa. On-chip integrated power management mppt controller utilizing cell-level architecture for pv solar system. Solar Energy, 117:10-28, 2015.

[15] Ping Li, Yumei Wen, Chaobo Jia, and Xinshen Li. A magnetoelectric composite energy harvester and power management circuit. IEEE Transactions on Industrial Electronics, 58(7):2944-2951, 2010.

[16] YC Shu and IC Lien. Analysis of power output for piezoelectric energy harvesting systems. Smart materials and structures, 15(6):1499, 2006.

[17] M Renaud, R Elfrink, M Jambunathan, C de Nooijer, $\mathrm{Z}$ Wang, M Rovers, R Vullers, and $\mathrm{R}$ van Schaijk. Optimum power and efficiency of piezoelectric vibration energy harvesters with sinusoidal and random vibrations. Journal of Micromechanics and Microengineering, 22(10):105030, October 2012.

[18] Elie Lefeuvre, Gael Sebald, Daniel Guyomar, Mickael Lallart, and Claude Richard. Materials, structures and power interfaces for efficient piezoelectric energy harvesting. Journal of Electroceramics, 22(1-3):171-179, February 2009.

[19] A Brenes, A Morel, J Juillard, E Lefeuvre, and A Badel. Maximum power point of piezoelectric energy harvesters: a review of optimality condition for electrical tuning. Smart Materials and Structures, 29(3):033001, 2020.

[20] Reinhilde D'hulst, Tom Sterken, Robert Puers, Geert Deconinck, and Johan Driesen. Power Processing Circuits for Piezoelectric Vibration-Based Energy Harvesters. IEEE Transactions on Industrial Electronics, 57(12):4170-4177, December 2010.

[21] Ali Koteiche, Alexis Brenes, Kevin Malleron, and Gerard Sou. Power-limiting phenomena in a magnetoelectric transducer for wireless power transfer. Wireless power transfer conference, 2020.

[22] Haruhiko Asanuma and Toshihiko Komatsuzaki. Nonlinear piezoelectricity and damping in partially-covered piezoelectric cantilever with self-sensing synchronized switch damping on inductor circuit. Mechanical Systems and Signal Processing, 144:106867, October 2020.

[23] Samuel C. Stanton, Alper Erturk, Brian P. Mann, and Daniel J. Inman. Nonlinear piezoelectricity in electroelastic energy harvesters: Modeling and experimental identification. Journal of Applied Physics, 108(7):074903, October 2010.

[24] Angela Triplett and D. Dane Quinn. The Effect of Nonlinear Piezoelectric Coupling on Vibration-based Energy Harvesting. Journal of Intelligent Material Systems and Structures, 20(16):1959-1967, November 2009.

[25] Marco Amabili. Nonlinear damping in nonlinear vibrations of rectangular plates: Derivation from viscoelasticity and experimental validation. Journal of the Mechanics and Physics of Solids, 118:275-292, September 2018.

[26] Christian Lalanne. Non-viscous damping. Sinusoidal Vibration, pages 261-289, 2014.

[27] Ashkan Haji Hosseinloo and Konstantin Turitsyn. Fundamental limits to nonlinear energy harvesting. Physical Review Applied, 4(6):064009, 2015.

[28] Kevin Malleron, Aurelie Gensbittel, Hakeim Talleb, and Zhuoxiang Ren. Experimental study of magnetoelectric transducers for power supply of small biomedical devices. Microelectronics Journal, 88:184-189, 2019. 
[29] Tuan Anh Do, Hakeim Talleb, Aurélie Gensbittel, and Zhuoxiang Ren. 3-d finite element analysis of magnetoelectric composites accounting for material nonlinearity and eddy currents. IEEE Transactions on Magnetics, 55(10):1-8, 2019.

[30] F. Yang, Y.M. Wen, P. Li, M. Zheng, and L.X. Bian. Resonant magnetoelectric response of magnetostrictive/piezoelectric laminate composite in consideration of losses. Sensors and Actuators A: Physical, 141(1):129135, January 2008.

[31] R D'hulst, Tom Sterken, P Fiorini, Robert Puers, and Johan Driesen. Energy scavengers: Modeling and behavior with different load circuits. In IECON 2007-33rd Annual Conference of the IEEE Industrial Electronics Society, pages 2169-2174. IEEE, 2007. 